ISP

\section{Grant Services Offered}

The absence of safe, reliable, and tax-free channels to send assistance to the former Soviet Union (FSU) and the Baltic has been a major stumbling block to donor organizations. However, the International Science Foundation (ISF) has been able to transfer over 80 MSUS in direct grants, material and travel support to scientists from the two regions. The ISF says that its mechanisms are uniquely capable of ensuring that critically needed assistance will have the greatest possible impact. It has announced a programme to facilitate assistance efforts undertaken by non-profit donor organizations in support of non-commercial scholarly research and education programmes in the FSU and the Baltic countries. The ISF infrastructure will be available to other organizations for a $10 \%$ fee (naturally, all direct costs associated with a donation must be paid by the donor organization). This infrastructure includes exemptions from personal income, value-added and customs taxes, which typically account for $40 \%$ of the value of a donation. For further information, contact: Grant Assistance Programme, International Science Foundation, 1054 31st St. NW, Suite 110 , Washington, D.C. 20007, USA.

(Tel.: +1-202-342-27 60; fax +1-202-342-27 65; e-mail: info@isf.org)

\section{$\mathrm{ICHIA}$}

\section{Dialogue Needed}

Making the most effective use of worldwide resources is a dominant theme in many fields of science. It is therefore not surprising that a framework for international cooperation has been set up in nuclear physics in the form of a committee of the International Union of Pure and Applied Physics (IUPAP) Nuclear Physics Commission. The committee - called the International Committee for High Intensity Accelerators (ICHIA) - has decided to make international cooperation its principle focus. H. Feshbach, Emeritus Professor at the Massachusetts Institute of Technology, who chairs ICHIA argued forcibly at the EPS Large Facilities Conference in Lausanne (12-14 September) that the missing component in today's essentially international scientific community is a dialogue between scientists and policy makers. To help generate the framework, ICHIA is inviting scientists and policy makers (defined as those in charge of nuclear programmes) to a meeting in Amsterdam on 8-9 December. One-half will be devoted to policy issues such as protocols, while the other half will present aspects at the frontiers of nuclear science which have the potential to produce general results of wide applicability, and indeed direct applications. For further information, contact: Prof. H. Feshbach, MIT, 77 Mass Ave., Cambridge, MA 02138, USA.

(Tel.: +1-617-253 48 21; fax +1-617-253 86 74)

\section{EUPRO}

Details for the European Union of Physics Research Organizations (EUPRO) given in the August Directory issue and in an item on facilities (June/July 1994) are incorrect. The Secretary (Dr. M. Brocken) can be found at $+31-30$ 923273 (tel.) and +31-30-94 6099 (fax).

\section{PHYSICS SURVEY}

A survey Physics in the European Union in the ' 80 's (available from M. Brocken, FOM, Postbus 3021, NL-3502 GA Utrecht; fax: +31$30-946099$ ) reports that during the 1980's the then European Community plus Sweden and Hungary contributed almost $25 \%$ of all physics publications and maintained a stable citation rate. Denmark was ranked first in most of the reviewed aspects.

\section{CORRECTIONS}

The Editor apologizes to The Institute of Physics for referring to the society as having 14000 members in an item dealing with the IOP's new headquarters (EN, June/July 1994). The IOP in fact has 18500 members.

The Consorzio INFN listed as an Associate Member in the August Directory issue is now called the National Institute for the Physics of Matter following a change in status.

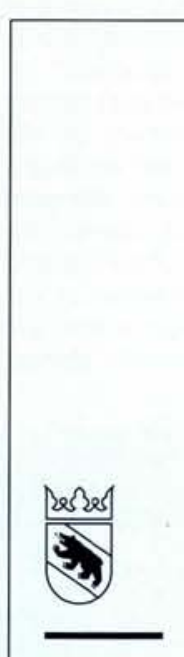

\section{UNIVERSITY OF BERN}

\section{FULL PROFESSOR OF PHYSICS}

The University of Bern invites applications for a full professorship of physics in the Physikalisches Institut, Department of Mass Spectrometry and Space Research. The position is open from October 1, 1996. Present fields of research in the Department include the origin and the evolution of the solar system (meteorites, comets, Moon, planets), the Sun and its influence on planets (solar wind, planetary atmospheres and magnetospheres, cometary comae), physics of the interstellar matter, and physics of the middle and of the upper atmosphere. The department offers an excellent infrastructure (staff, laboratories, equipment) for mass spectrometric analyses in the laboratory and for in-situ investigations in space. Experimental research activities involve the development and construction of mass spectrometers of different types. Ideal age of the applicants: 30 to 45 . Candidates with a record of successful research and who are prepared to participate in the teaching and administrative duties of the Institute are invited to submit their applications. including curriculum vitae, list of publications and a research programme, by March 1,1995 to the Erziehungsdirektion des Kantons Bern, Amt für Hochschulen (1600.40/94), Sulgeneckstrasse 70, CH-3005 Bern, Switzerland.

Further information can be obtained from the

Acting Director of the Physikalisches Institut,

Prof. H. Balsiger, Sidlerstrasse 5, CH-3012 Bern, Switzerland, Tel: +41316314414, Fax: +41316314405

\section{Climate and Environmental Physics \\ Physics Institute, University of Bern (Switzerland)}

has an opening for a

\section{Tenure Track Position in Environmental Physics and/or Global Change Research}

We are seeking a creative and independent scientist with extensive experience in one of the following specialities: (i) environmental physics using modern measurement techniques with an emphasis on stable isotopes, (ii) reconstruction of global and regional climate change using isotope analysis in paleoclimatic archives, (iii) modelling of biogeochemical cycles (especially the carbon cycle including its isotopes) on time scales of up to $10^{6}$ years.

A PhD in physics, environmental sciences or a related discipline and post-doctoral experience of at least 2 years are required. Development of additional own research projects in this field and participation in the teaching duties of the Physics Institute are expected. Appointment is on the level of "Hochschulassistent-in" or "Hochschuloberassistent-in" depending on experience and is for 3 years initially, starting from January 1995 or later. The position is renewable up to 7 years. The promotion to tenure requires a Habilitation (i.e. track record of publications and active research).

Please send a statement of your current research interests and experience, curriculum vitae with list of refereed publications, copies of your three most important papers, and the name addresses, phone and fax numbers of three referees to Prof. Thomas F. Stocker, Physics Institute, University of Bern, Sidlerstrasse 5, 3012 Bern, Switzerland. The University of Bern is an equal opportunity/affirmative action employer. 